\title{
On representing information: a characterization of the analog/digital distinction
}

\begin{abstract}
The common account of the analog vs digital distinction is based on features of physical systems, being related to the usage of continuous vs discrete supports respectively. It is proposed here to alternatively characterize the concepts of analog and digital as related to coding systems, of which a formal definition is given, by suggesting that the distinction refers to the strategy adopted to define the coding function: extensional in digital systems, isomorphic intensional in analog systems. This thesis is supported by examples, in particular of analog systems exploiting discrete supports, and is discussed so to explain why digital coding is currently so widespread in the technological and social practice.
\end{abstract}

KEYWORDS: analog; digital; information; coding system; extensional representation; intensional representation.

\section{Introduction}

The information acquired in interacting with the world is exploited by encoding it by means of signs recorded on more or less stable physical supports. As widely acknowledged, the analog vs digital distinction ("A/D distinction", from now on) pertains to the way the processes of encoding and recording are performed. ${ }^{1}$ Still, while the ways of encoding and recording are acknowledged as depending on both the linguistic level of signs and the ontological level of that which signs refer to, less emphasized is the relevance of the relation, i.e., the code, that links the two levels. This multifaceted subject is not new in philosophy, and its relevance has been acknowledged since the works by N. Goodman (1968) and D. Lewis (1971).

This paper is aimed at giving a new account of the A/D distinction, construed as pertaining to the kind of code exploited to perform the encoding process. ${ }^{2}$ This account is intended to provide a descriptive definition of the distinction enabling us both to cover the paradigmatic cases to which the distinction applies and to meet some basic desiderata stemming from the analysis of the theories proposed to classify such cases. The thesis we propose can be synthesized as follows: a code can be interpreted as a function linking signs and information entities, and the way of defining the code determines whether the representation is analog or digital. In particular, if the code function is defined extensionally (i.e., by a list), the representation is digital, whereas if it is defined intensionally (i.e., by a rule), the representation is analog.

According to such a characterization the concepts 'analog' and 'digital' pertain to processes involving information, but their specific scope is delimited to the complementary stages of encoding and decoding: what is analog or digital is the representation of information. The option of extending the attribution, thus possibly talking about analog or digital description, analog or digital explanation, analog or digital computation, .... is understood as a rhetorical move to refer to description, explanation, computation, ... related to information represented as analog or digital.

The rest of the paper is devoted to introduce and defend this proposal, and is articulated as follows. Section 2 highlights some common intuitions about the A/D distinction and proposes two basic desiderata on a possible interpretation of the distinction. Section 3 briefly discusses the concepts of classification system, and information together with the derived concept of coding system, on which the A/D distinction is grounded: this establishes our basic terminology. Section 4 critically analyzes a number of views concerning

\footnotetext{
${ }^{1}$ As it will be discussed in Section 3, a common understanding relates the A/D distinction to the continuity or discreteness of the physical system exploited as support in the encoding process. However, dissenting opinions have already been put forward on this matter: Lewis (1971) focused the A/D distinction on information encoding, instead of on features of the physical support, and his view has been recently further developed by Maley (2011). Although this is a definite improvement over the received view, the present paper will try to show that both Lewis' and Maley's positions are only in part correct. In particular, we believe that Maley's characterization of analog is substantially correct, although not sufficiently general, but we dissent from his characterization of digital as the encoding of numbers by means of a positional system.

2 The reference of the A/D distinction to information encoding allows including the distinction between analog and digital computation (see, e.g., Minsky (1972)) as a derived case, under the intuitive assumption that A/D computation is a process operating on A/D coded entities. On the other hand, this admittedly excludes all purely functional and a-semantic processes from the present analysis and proposal. For example, Piccinini and Scarantino (2011) adopt the A/D distinction as referred to computation defined as "the processing of vehicles according to rules that are sensitive to certain vehicle properties and, specifically, to differences between different portions of the vehicles" (p.10). According to them, in digital computation "strings of discrete elements" (p.7) are manipulated, whereas "analog computers are systems that manipulate continuous variables to solve certain systems of differential equations. Continuous variables are variables that can vary continuously over time and take any real values within certain intervals" (p. 11).
} 
the $\mathrm{A} / \mathrm{D}$ distinction as presented in technological and philosophical fields. On this ground in Section 5 the proposed definition is finally introduced and commented. Section 6 contains some concluding remarks.

\section{Basic intuitions}

The A/D distinction is typically applied to the way in which information is represented by means of signs or signals and, by extension, to systems implementing processes involving signs or signals such as clocks, discs, cameras, computers. The quantitative representation of the motion of a runner as a function of time exemplifies the basic features of the A/D distinction.

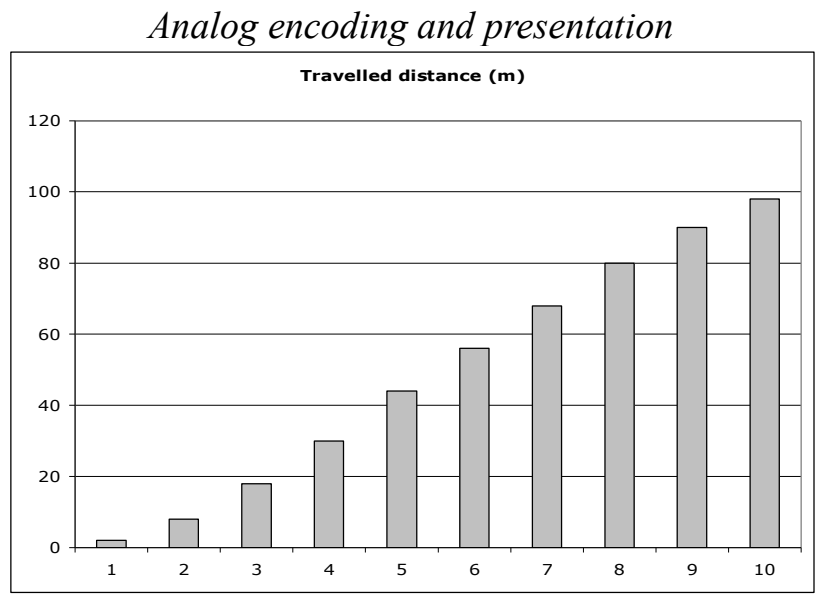

Digital encoding and presentation
\begin{tabular}{|c|c|}
\hline Time (s) & Traveled distance $(\mathrm{m})$ \\
\hline 1 & 2 \\
\hline 2 & 8 \\
\hline 3 & 18 \\
\hline 4 & 30 \\
\hline 5 & 44 \\
\hline 6 & 56 \\
\hline 7 & 68 \\
\hline 8 & 80 \\
\hline 9 & 90 \\
\hline 10 & 98 \\
\hline
\end{tabular}

In the analog encoding the heights of the histogram bars are proportional to the traveled distances: the histogram is drawn by establishing a covariant relation, in fact a morphism, between the signs and what they codify. In addition, since the traveled distance is typically interpreted as a continuous quantity, the histogram bars representing it may have in principle continuously varying heights. In the digital encoding such similarity disappears and the signs, sequences of digits in this case, are linked to the encoded entities by a conventional relation. These considerations can be extended by considering the following paradigmatic cases of representation of information:

(1) positional representation of positive integer numbers;

(2) positional representation of integer numbers;

(3) positional representation of rational numbers (e.g., $-0.5,-0.2,0,0.2,0.5$ );

(4) fractional representation of rational numbers (e.g., $-1 / 2,-1 / 5,0,1 / 5,1 / 2)$;

(5) representation of time by means of a sand clock;

(6) representation of time by means of a clock with dials;

(7) representation of time by means of a clock with digits;

(8) representation of music by means of a vinyl disk;

(9) representation of music by means of a compact disc;

(10) representation of a quantity by means of a histogram on paper;

(11) representation of a quantity by means of a histogram on PC screen;

(12) representation of a quantity by means of a table of numbers on paper or PC screen.

Cases (5), (6), (8), (10), (11) are typically acknowledged as analog representations, based on a similarity, or analogy, between what represents and what is represented. Conversely, cases (1), (2), (3), (4), (7), (9), (12) are typically classified as digital representations, exploiting numerical digits as means to encode the entities to be represented according to a rule that is only conventional.

A preliminary classification of these examples is thus the following:

\begin{tabular}{|l|l|l|}
\hline PRELIMINARY CLASSIFICATION & classified cases & non classified cases \\
\hline analog representation & $(5),(6),(8),(10),(11)$ & none \\
\hline digital representation & $(1),(2),(3),(4),(7),(9),(12)$ & none \\
\hline
\end{tabular}


In light of the previous examples, the following traits seem to be characteristic of analog or digital coding respectively:

Analog coding:

A1. a similarity relation between the signs and the information entities they codify;

A2. signs used to codify such that a similarity representation is possible.

Digital coding:

D1. a conventional relation between the signs and the information entities they codify;

D2. signs used to codify that are discrete and (can be in one-to-one correspondence with) sequences of digits.

For example, the histogram representation in the Figure above is consistent with A1 and A2, since information is represented by exploiting a similarity, or analogy, between what represents and what is represented. On the other hand the table is consistent with D1 and D2, since information is represented by using numerical digits that are conventionally related to what is to be represented.

In what follows we will use these cases, and the above preliminary classification, together with traits A1, A2, D1, and D2, as a sort of empirical basis to assess theories. In particular, the classification will allow us to argue on the theory to be preferred in a context in which competing theories $\mathrm{T}_{i}$ are available, according to the following two desiderata:

Desideratum 1: domain completeness.

$\mathrm{T}_{i}$ is to be preferred to $\mathrm{T}_{j}$, if $\mathrm{T}_{i}$ has a broader scope, i.e., classifies more cases, than $\mathrm{T}_{j}$.

Desideratum 2: intuition consistency.

$\mathrm{T}_{\mathrm{i}}$ is to be preferred to $\mathrm{T}_{j}$, if $\mathrm{T}_{i}$ is consistent with the preliminary classification and $\mathrm{T}_{j}$ is not.

Hence, the preliminary classification is not used to unconditionally rule out a theory, but only in a ceteris paribus situation, i.e., if the theory does not have a broader scope than its alternative(s). In addition, the intuition on which Desideratum 2 is based is supported not only by the above considerations but also by the fact that most of the accounts we are going to survey converge on the given preliminary classification.

\section{Assumptions on information and coding systems}

We assume here that the basic structure of information acquisition and communication can be conceived as follows: ${ }^{3}$

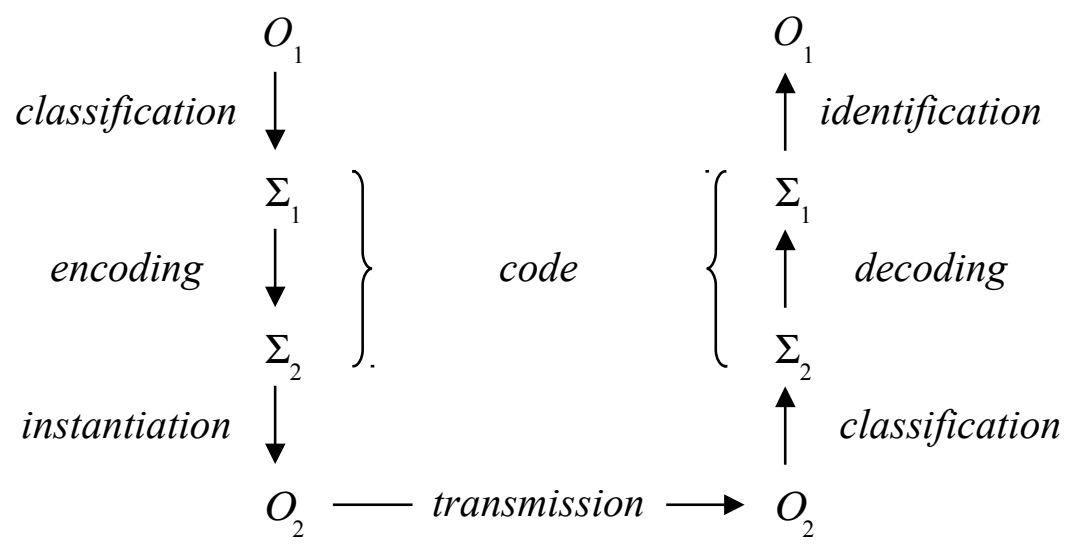

The idea is that the process of acquisition of information on a set $O_{1}$ of entities is a process of classification of such entities into a set $\Sigma_{1}$ of abstract classes, which in virtue of a code is linked to a set $\Sigma_{2}$ of abstract

\footnotetext{
${ }_{3}^{3}$ This assumption is consistent with almost every account of the structure of information and of communication process we are aware of (see Adriaans \& van Benthem (2008), ch. 1 for a general introduction on the subject). In particular, it is consistent with Floridi's characterization of information (cfr. Floridi (2011a) and (2011b)). What he calls data correspond to our set $O_{1}$ while his levels of abstraction correspond to possibly different sets of abstract objects. The picture is presented to highlight that such a process is essentially based on an encoding function linking abstract entities such as information contents and signs intended as types. This aspect is often not explicitly acknowledged in standard presentations. For a compact introduction see Bremer and Cohnitz (2005).
} 
signs. Abstract signs are then instantiated by concrete signs in $\mathrm{O}_{2}$, thus said to convey information about entities in $O_{1}$. Such concrete signs can be transmitted and interpreted by an agent who / which is able to identify the entities in $O_{1}$ as elements of the classification $\Sigma_{1}$ by means of her / its knowledge of the code, so that the information on the entities in $O_{1}$ is communicated by transmitting the entities in $O_{2}$.

Let us introduce some definitions to setup the conceptual framework. As an example, suppose that an object (an element of $O_{1}$ ) is classified as a book instead of some other object, where being a book is an abstract property (an element of $\Sigma_{1}$ ). Hence, that the object is a book is a piece of information, that can be coded by some signs (elements of $\Sigma_{2}$ ), types which are instantiated in concrete tokens (elements of $O_{2}$ ). These tokens can be transmitted to an addressee. In order to decode the message the addressee must trace back the tokens of the symbols to their types and then extract the piece of information coded by them.

\subsection{Classification systems}

Information is basically linked to classification. ${ }^{4}$

Definition of classification system.

A classification system is a triple $\mathbf{S}=\langle O, \Sigma, f\rangle$, where:

i) $O$ is a set

ii) $\Sigma$ is a set

iii) $f$ is a function, called a classification function, from $O$ to $\Sigma$

$O$ is a set of objects $^{5}$ (also referred to as tokens), i.e., objects under classification. $\Sigma$ is a set of sorts (also referred to as types), under which the objects of $O$ are to be classified by means of the classification function $f \cdot{ }^{6}$

Definition of coding system.

A coding system is a triple $\mathbf{C}=\left\langle\Sigma_{1}, \Sigma_{2}, f_{\mathrm{C}}\right\rangle$, where:

i) $\Sigma_{1}$ is the range of a classification function, i.e., is such that there exists a classification system $\mathbf{S}=$ $\left\langle O_{1}, \Sigma_{1}, f_{1}\right\rangle$

ii) $\Sigma_{2}$ is a set of abstract objects (abstract signs), whose instances are producible and observable

iii) $f_{\mathrm{C}}$ is a one-to-one function, called a coding function, from $\Sigma_{1}$ to $\Sigma_{2}$

By i), a coding system operates on the sorts which classify the objects of a set. By ii), the classifying objects have both actually producible (i.e., it must be possible to write them) and observable instances (i.e., it must be possible to read them). Finally, by iii), a coding function must be invertible, so that decoding, as formalized by the mapping $f_{\mathrm{C}}^{-1}$, generates unambiguous outputs. ${ }^{7}$

\section{Definition of information.}

\footnotetext{
${ }^{4}$ See Barwise and Seligman (2007), part 2, and Bremer and Cohnitz (2005), ch. 4. To our knowledge, the first extensive use of the concept of classification for modeling information and information flow is due to Barwise (1989).

5 "Object" is used here in a broad sense, as synonymous of "entity", so that events, situations, facts and concepts are all objects.

${ }^{6}$ In the definition above no constraints have been introduced on the cardinality of the sets $\Sigma$. An objection might be that a classification is useful only if its classes can be listed, and therefore $\Sigma$ is finite or at most denumerable. On the other hand, the A/D distinction, as characterized here, does not depend on this requirement, so that any further specification on the definition of a classification system remains just an option.

${ }^{7}$ An anonymous referee objected that in principle the definition should be applicable to cases of ambiguous signs such as words of natural languages. There are many ways to treat ambiguity in natural languages, one of which entails a minimal depart from the framework sketched here. According to this treatment, ambiguity involves two different words having two different meanings but the same phonetic (and/or graphic) form. For instance, in English there are two words ("bank 1 " and "bank 2 ") having the same form, the first meaning "financial institution" and the second "raised area along the side of a river". Dictionaries usually presuppose this treatment of ambiguous words. The coding and decoding functions take as arguments not "bank", but "bank " or "bank" and this guarantees unambiguous outputs. Of course, when an ambiguous word is decoded a preliminary process is in order, which must determinate whether the word to be decoded is "bank 1 " or "bank 2 ". However, this process is usually not considered semantic but pragmatic. Sometimes processes which precede semantic decoding are called presemantic (for example, see Perry (1997)). Were ambiguity allowed as non-injectivity of the coding function, a generalized concept of weak coding system would be possibly obtained, whose exploration is outside the scope of this paper.
} 
Let $\mathbf{S}=\left\langle O_{1}, \Sigma_{1}, f_{1}\right\rangle$ be a classification system, such that the classification function $f_{1}$ is not a constant, i.e., there exist $o_{i}, o_{j} \in \Sigma_{1}, o_{i} \neq o_{j}$, such that $f_{1}\left(o_{i}\right) \neq f_{1}\left(o_{j}\right)$. The object $f_{1}(o) \in \Sigma_{1}$ is said to be a piece of information about the object $o \in O_{1}$ with respect to $\Sigma_{1}$, and $\mathbf{S}$ is said an informative classification system.

This concept of information is grounded on the minimal requirement that something, $y$, is informative on something, $x$, if from $y$ some difference on $x$ can be inferred. Indeed, $f_{1}\left(o_{i}\right)$ is informative on $o_{i}$ because there must be at least one $o_{j}$ such that $f_{1}\left(o_{i}\right) \neq f_{1}\left(o_{j}\right)$. Hence from $f_{1}\left(o_{i}\right)$ the inference 'the object classified by $f_{1}$ is not $o_{j}^{\prime}$ is obtained. ${ }^{8}$

\section{Definition of conveying information.}

Let $\mathbf{C}=\left\langle\Sigma_{1}, \Sigma_{2}, f_{\mathrm{C}}\right\rangle$ be a coding system. The object $\sigma \in \Sigma_{2}$ is said to convey information on an object $o \in O$ if and only if there exists an informative classification system $\mathbf{S}=\left\langle O, \Sigma_{1}, f_{1}\right\rangle$ such that $\sigma=f_{\mathrm{C}}\left(f_{1}(o)\right)$.

Since $f_{\mathrm{C}}$ is invertible, $f_{\mathrm{C}}^{-1}(\sigma)=f_{1}(o)$; hence, by decoding $\sigma$, the class in $\Sigma_{1}$ to which $o$ belongs in virtue of $f_{1}$ is known. In this way, the previously introduced picture is wholly clarified.

On this basis the $\mathrm{A} / \mathrm{D}$ distinction can now be discussed.

\section{The customary understanding of the $A / D$ distinction}

Both the customary and the philosophical interpretation of the A/D distinction seem to be strongly influenced by the way the distinction is understood in the theories of control systems and signals processing, where the following general positions are, often implicitly, assumed: 1) 'being analog' is related to the continuity of physical supports, or of the mathematical model of them; 2) 'being digital' is related to the discreteness of physical supports, or of the mathematical model of them.

The definitions proposed by a specifically authoritative source in the technological field, the International Electrotechnical Vocabulary (IEV) resulting from the standardization activities of the International Electrotechnical Commission (IEC), seem to follow this interpretation. Given that (see IEV 351-21-53 and IEV 351-21-54):

(i) an analog signal is a signal whose information parameter is a physical quantity which may assume any value within a given range at any instant within a continuous time interval

(ii) a digital signal is signal whose information parameter may assume one out of a set of discrete values within a given range ${ }^{9}$

the following definitions are proposed:

analog $:=$ pertaining to the representation of information by means of an analog signal.

(see IEV 101-12-05)

digital := pertaining to the representation of information by means of a digital signal.

(see IEV 101-12-07)

The table shows the classification of the examples (1)-(12) mentioned in Section 2 according to these definitions:

\begin{tabular}{|l|l|l|}
\hline IEV CLASSIFICATION & classified cases & problematic cases \\
\hline analog representation & $(6),(8),(10)$ & \multirow{2}{*}{ none } \\
\cline { 1 - 2 } digital representation & $(1),(2),(3),(4),(5),(7),(9),(11),(12)$ & \\
\hline
\end{tabular}

\footnotetext{
8 This is consistent with the well known characterization proposed by Bateson (1979) "All receipt of information is necessarily the receipt of news of difference" (p.29). More formally, the definition of information entropy by Shannon (1948) shows that for a source to convey information at least two objects, such that their probabilities of being communicated is positive, must be present.

${ }^{9}$ For a more analytical presentation see also Allen \& Mills (2004, Chapter 1).
} 
In most cases the classification following from IEV definitions is in agreement with the intuitive classification introduced above. The cases that are not in agreement are those in which, although the representing signals and the represented objects covary, signals are not continuous. Indeed, in case (5) (representation of time by means of sand-glass) and in case (11) (representation of a quantity by means of a diagram on a PC) the representations have no constraints but covariance, yet signals are clearly discrete.

One might expect that philosophical theories of the A/D distinction built on the opposition between discrete and continuous signals would be consistent with the IEV. As we will see in the following section, this is not the case.

\subsection{Positions centered on the continuous vs discrete opposition}

Let us consider first the positions according to which the continuous vs discrete opposition is crucial in order to understand the $\mathrm{A} / \mathrm{D}$ distinction.

\subsubsection{Goodman's account}

According to Goodman (1968), the A/D distinction is outlined by the following definitions.

Goodman's definition of analog: a system is analog if syntactically and semantically dense (p. 160), where a system is (1) syntactically dense if it consists of an infinite set of ordered symbols such that for each pair of symbols $a$ and $b$ such that $a<b$, where $<$ is the order defined on the system, there is a symbol $c$ in the set such that $a<c<b$, and (2) semantically dense if it consists of an infinite set of ordered symbols, each symbol denotes a class of objects, and the denoted classes are in their turn infinite and ordered, so that for each pair of such classes there is a third class included between them with respect to the given order. ${ }^{10}$

Goodman's definition of digital: a system is digital if it is syntactically and semantically discontinuous and differentiated, where a system is: (1.1) is syntactically discontinuous if it is not syntactically dense; (1.2.) syntactically differentiated if it is always theoretically possible to determine whether a given token belongs to a given symbol type; (2.1) semantically discontinuous if it is not semantically dense; (2.2) semantically differentiated if it is always possible to determine whether a given token belongs to a given type. ${ }^{11}$

The A/D distinction, as characterized by Goodman, can be straightforwardly expressed in terms of the process of information acquisition and encoding, as introduced in Section 2: in an analog system the types of symbols $\Sigma_{2}$ are densely ordered, while in a digital one they are not. Accordingly, in digital systems it is possible to determine of which object of $\Sigma_{2}$ a token of $\mathrm{O}_{2}$ is an instance, whereas in analog systems this possibility is not assured. Furthermore, $\Sigma_{1}$ represents the semantic level of the objects used in the encoding, i.e., it contains the entities denoted by such classes. Again, a system is analog only if the objects of $\Sigma_{1}$ are densely ordered, while in a digital system they are not. As a consequence, Goodman argues that analog and digital systems have nothing special to do with digits (or numerals) and analogy respectively. As we shall see in Section 5.3, we agree with Goodman on the first point but not on the second one, since, more generally, we disagree with the views which identify continuous with analog and discrete with digital.

\subsubsection{Some problems in Goodman's account}

The examples of Section 2 are classified as follows according to Goodman.

\begin{tabular}{|l|l|l|}
\hline GOODMAN CLASSIFICATION & classified cases & problematic cases \\
\hline analog representation & $(3),(4),(6),(8),(10)$ & \multirow{2}{*}{$(5),(7),(9),(11),(12)$} \\
\cline { 1 - 2 } digital representation & $(1),(2)$ & \\
\hline
\end{tabular}

\footnotetext{
${ }^{10}$ An example of a system that is analog according to this definition is an ideal dynamometer, whose indicating needle changes position as the applied force changes. Indeed, for each pair of positions of the needle there is, in principle, an intermediate position that the needle can assume, and, if classes of objects having the same weight are taken into account, then for each pair of such classes of objects there is a third class of objects of intermediate weight corresponding to such an intermediate position.

${ }^{11}$ An example of a system that is digital according to this definition is a device that reports the numbers of coins that have been inserted in it after the last reset, the count being expressed by a sequence of characters so that, e.g., "****" stands for 'three coins'. Indeed, the characters are syntactically discontinuous and differentiated and every set of coins is distinguishable from the others.
} 
A problem of Goodman's analysis is the incompleteness of the classification following from his criteria: assuming a dense order on $\Sigma_{1}$ and $\Sigma_{2}$ in the analog representation and a non-dense order in the digital one, he excludes the cases in which only one of sets is densely ordered from his classification. Consider the example (7) (representation of time by means of a watch with digits). While instants of time are customarily supposed to be densely ordered, watch digits are not so ordered. Therefore, in this case, $\Sigma_{1}$ is densely ordered, while $\Sigma_{2}$ is not, with the consequence that this example is neither analog nor digital according to Goodman's definition. Similar remarks apply to examples (5), (9), (11) e (12): Goodman's criteria are in contrast with Desideratum 1. Moreover, another consequence of Goodman's account is that examples (3) and (4) (the representations of rational numbers by means of positional and fractional systems) are classified as analog, given that both the representing and the represented systems can be densely ordered. However, the preliminary classification, IEV and most of proposed classifications regard these cases as digital: Goodman's criteria are in contrast also with Desideratum 2.

Finally, despite the negation explicitly stated by Goodman, his position assumes both a potentially infinite resolution of the indicating instruments and observations, to guarantee the condition of syntactical density, and a potentially infinitesimal variability of the property under consideration, to guarantee the condition of semantic density. This implies that analog systems cannot be based on indicating instruments of discrete resolution nor on discretely varying properties. While the issue of the continuity or discreteness of properties is a matter of physics, and thus outside the present analysis, the very concept of infinite resolution for a technological device is critical: endorsing it as a necessary condition for a system to be analog leads to the conclusion that, strictly speaking, no empirical system can be actually analog.

\subsubsection{Haugeland's account}

Haugeland (1981) suggests that continuity and discreteness are primarily relevant as properties not of the representation support, as supposed by Goodman, but of the instancing (i.e., writing and reading) process on the support. Thus, although water is considered continuous according to macroscopic physical models, a given amount of water can be exploited as a discrete support to convey information. ${ }^{12}$ On the other hand, even if sand is considered as discrete, a given amount of sand can be dealt with as a continuous support. ${ }^{13}$ On this basis, Haugeland believes that the A/D distinction concerns the procedures of writing and reading tokens of various types, i.e., "the procedures for producing tokens, given the types that they are supposed to be, and the procedures for telling and determining the types of given tokens": these procedures succeed without margin for error in the case of digital devices but do not offer similar guarantee in the case of analog devices. Accordingly, the definitions given by Haugeland are the following.

Haugeland's definition of digital: a device is digital if the process it exploits for writing and reading tokens of a given type can be guaranteed to be positive and reliable, where such a process (i) is positive if it certainly succeeds, i.e., its writing/reading results can be unambiguously assessed, and (ii) is reliable if it always succeeds, provided that the process itself is performed under appropriate conditions.

Haugeland's definition of analog: a device is analog if the process it exploits for writing and reading tokens of a given type cannot be guaranteed to be positive and reliable, i.e., only approximated results can be obtained from it.

A customary origin of the unavoidable errors that cause the approximation to which Haugeland refers is the adoption of sets of tokens that are dense in the Goodman sense. If such tokens are not "sufficiently distinct", so that between each pair of tokens there is a third one that could not be operatively distinguishable from either of them, the attribution of a token to a given type can indeed be only approximately obtained. If Goodman's and Haugeland's accounts of the A/D distinction are in this sense similar, some differences remain between their positions. According to Haugeland, the distinction relates to the process of writing and reading tokens, not to the structure of the set of tokens. Therefore, for example, a device that might produce a very large range of electrical tensions but that in fact is designed to generate only signals close to $0 \mathrm{~V}$ or to 5

\footnotetext{
$\overline{12}$ Consider, for example, a system using a supply of water and a supply of beakers. Water is poured into the beakers in equivalently-sized increments, such that each beaker contains between zero and nine increments of water. There is a one-to-one function associating beakers with the numbers zero through nine, such that the amount of water in a beaker determines the number with which that beaker is associated (cfr. Katz (2008), p. 404). In this case water is used as a discrete support.

${ }^{13}$ Consider, for example, how sand is exploited in hourglasses in order to measure time intervals.
} 
V, i.e., two "sufficiently distinct" sets of signals, would be considered digital by Haugeland because, under suitable conditions, a positive and reliable decoding process operating on such tensions is available. On the other hand, Goodman would characterize it as analog since there is no clear-cut distinction between the two types, so that, for example, an ambiguity remains on the type corresponding to a $2.5 \mathrm{~V}$ tension, that is physically possible. Hence, according to Haugeland, the A/D distinction relates to the relation between the sets $\Sigma_{2}$ and $O_{2}$, as realized by the writing and reading process. Since, against the Goodman's hypothesis, Haugeland does not introduce any condition on $\Sigma_{1}$, his position is a purely syntactic one, involving only the relations between types and tokens.

Katz (2008) takes up a position very similar to Haugeland's. While agreeing that in digital systems tokens are sufficiently far from one another to prevent misreadings, he stresses that such a concept of sufficiency is of a pragmatic nature, as it should be characterized relatively to users and their goals.

\subsubsection{Some problems in Haugeland's account}

The examples of Section 2 are classified as follows according to Haugeland.

\begin{tabular}{|l|l|l|}
\hline HAUGELAND CLASSIFICATION & classified cases & problematic cases \\
\hline analog representation & $(6),(8),(10)$ & \multirow{2}{*}{$(5),(11)$} \\
\cline { 1 - 2 } digital representation & $(1),(2),(3),(4),(7),(9),(12)$ & \\
\hline
\end{tabular}

Haugeland's accounts is consistent both with the preliminary classification and with IEV. Only two problems remain. First, examples (5) and (11) are problematic because it is not clear how Haugeland would consider them: when sand glasses and histograms on a PC are concerned, it may be asked if the system that writes and read tokens is the human agent using sand glasses and PCs or not. PCs discriminate between two histograms even if they differ for one pixel only, but human eye might not. The two systems allow a reliable reading in principle, but this is not their typical use. Second, Haugeland does not seem to account for the condition of being based on, or related to, an analogy, i.e., the resemblance between signs and represented objects, of analog systems. The following examples illustrate this issue.

Example 1: In some watches the minute hand does not move continuously, but jumps to the next notch each minute. Hence, the display has 60 discrete states. However, this does not change the common understanding that these watches are analog systems. On the other hand, according to Haugeland's criteria they should be considered digital, because the classes derived from them (i.e., the angular position of the hand at each instant of time) can be positively and reliably ascribed to such classes.

Example 2: In the dashboard of some vehicles, the fuel level is displayed by means of a sequence of LEDs (i.e., on/off light elements), such that when the tank is full all elements are on, and the number of on elements decreases as the tank empties. Despite the usage of an array of bistable entities, this system is similar to the one of the previous example and therefore it could be considered analog. Yet it encodes the information regarding the fuel level as discrete.

Example 3: Blachowicz (1997) argues about a slide rule whose cursor can only be located at certain points of the basic scale (there could be click-stops for such alignments). Slide rules are prototypical analog devices, and such a device would still function as a slide rule. Nevertheless, the cursor could only be in a finite number of discrete positions.

These examples share a feature: there is a covariance between the signs and what the signs represent and there is no further constraint on the signs themselves. In particular, the representation system makes use of discrete signs sufficiently distinct with each other to make the reading and writing processes positive and reliable. To sum up, the Haugeland's account is interesting and the classification it engenders is sufficiently intuitive, but still it does not satisfactorily covers all aspects of the A/D distinction. An alternative account might fare better if and only if (1) it is more intuitive and (2) is able to explain why Haugeland's view succeeds in the cases above. We will see that our proposal meets both these requisites. 


\subsection{Positions not centered on the continuous vs discrete opposition}

Let us consider now the positions according to which the continuous vs discrete opposition is not crucial in order to understand the $\mathrm{A} / \mathrm{D}$ distinction.

\subsubsection{Lewis'account}

Lewis (1971) is well aware that Goodman's hypothesis is problematic because there are many non-dense and differentiated representations which, however, are considered to be analog. For example, an integer number $n$ can be represented by a sequence of $1 \Omega$ electrical resistors connected in series in a circuit in which a switch allows the connection of exactly $n$ of them, thus obtaining an equivalent resistance of $n \Omega$. Lewis maintains that the A/D distinction is related to the physical quantity (he uses "magnitude") chosen as the representation means: if it is a "primitive or almost primitive" quantity, then the representation is analog; if, on the other hand, such a quantity is defined as a function of other (primitive or non-primitive) quantities, then the representation is digital. In addition, whether a quantity is primitive or not depends, according to Lewis, on the current state of knowledge of physics. In the example above, the representation of integer numbers by means of the given set of resistances is analog because a primitive quantity, the electrical resistance, is directly exploited to represent such numbers.

Let us consider, instead, a device constituted of several electric circuits, each of them with a resistance of either $5 \Omega$ or $1 \Omega$. Let the circuits be independent and ordered according to an index $i$ from 0 to $n$, all of them being connected to an output component that computes the number $\Sigma m_{i} 2^{i}$, where $m_{i}$ is either 0 or 1 depending on the resistance of the $i$-th circuit. For example, to represent the number 6 , three independent circuits are required, numbered 0,1 and 2 . The circuit 0 must have resistance $1 \Omega$, the circuits 1 and 2 resistance $5 \Omega$. The represented number is $0 \cdot 2^{0}+1 \cdot 2^{1}+1 \cdot 2^{2}=6$, thus a realization of the familiar binary system. In this case, the representation is digital, as it is the function of many differentiated primitive physical quantities, i.e., the resistances of different independent circuits.

Hence, Lewis claims that the A/D distinction concerns the relations between the sets $\Sigma_{1}$ and $\Sigma_{2}$ in a coding system. If the elements of $\Sigma_{2}$ are quantities obtained from the same determinable primitive property, and each of them directly represents an element of $\Sigma_{1}$, then the system is analog. On the contrary, if the represented elements depend on several elements of $\Sigma_{2}$, each of them obtained from one or more determinable properties, then the system is digital.

\subsubsection{Some problems in Lewis'proposal}

In Section 5 we will also argue that the A/D distinction relates to coding systems, i.e., to the relation between the sets $\Sigma_{1}$ and $\Sigma_{2}$. Therefore, we consider the Lewis' hypothesis to be an important improvement with respect to other positions because it identifies the right point where the matter must be settled. However, Lewis' proposal raises some critical issues.

The examples of Section 2 are classified as follows according to Lewis.

\begin{tabular}{|l|l|l|}
\hline LEWIS CLASSIFICATION & classified cases & non classified cases \\
\hline analog representation & $(5),(6),(8),(10),(11)$ & \multirow{2}{*}{$(1),(2),(3),(4),(7),(12)$} \\
\hline digital representation & $(9)$ & \\
\hline
\end{tabular}

Lewis seems to consider only the case in which numbers are represented by physical quantities, whereas the scope of the A/D distinction is much wider, as it could be generally related to information coding strategies. As an example, the number 5 may be represented by the numeral " 5 " and then instantiated by means of properly shaped ink spots on paper, but this can hardly be considered a case in which a number is represented by a physical quantity. Lewis' account does not satisfy the Desideratum 1 because examples (1), (2), (3), (4), (7) remain unclassified. In all these cases a number is represented by a numeral system. For the same reason numerical tables can hardly be considered a physical quantity and therefore also example (12) is not covered by Lewis' proposal. Second, Lewis claims that analog systems represent information by means of "primitive or almost primitive" quantities, while digital ones do so by the concurrence of several differentiated quantities. As already noted by Haugeland, this does not seem to be the case. An analog signal might be obtained by combining two different quantities, for example, positions of a pointer with sounds. It could be 
the case of an alarm signal operating so that when a pointer reaches successive given positions, higher and higher pitched sounds are emitted. The fact that two different quantities are involved in this signal does not make it digital.

\subsubsection{Maley's account}

Maley (2011) has refined Lewis' hypothesis. According to him, "analog representation is representation in which the represented quantity covaries with the representational medium, regardless of whether the medium is continuous or discrete. This is similar to Lewis' definition of analog representation, but without the requirement that the medium of representation be "primitive or almost primitive" (p. 6). His claim can be interpreted within the framework introduced in Section 2.

Maley's definition of analog: A representation $f_{\mathrm{C}}$ is analog if and only if (i) $\Sigma_{1}$ and $\Sigma_{2}$ are sets of quantities, on which additive operations $\bullet_{1}$ and $\bullet_{2}$ are defined respectively, and (ii) $f_{\mathrm{C}}: \Sigma_{1} \rightarrow \Sigma_{2}$ is such that for every two sorts $\sigma_{1}, \sigma_{2} \in \Sigma_{1}, \sigma_{1} \bullet \bullet_{1} \sigma_{2}=\sigma_{3}$ if and only if $f_{\mathrm{C}}\left(\sigma_{1}\right) \bullet_{2} f_{\mathrm{C}}\left(\sigma_{2}\right)=f_{\mathrm{C}}\left(\sigma_{3}\right)$, i.e., $f_{\mathrm{C}}$ is a homomorphism from $\left(\Sigma_{1}, \bullet_{1}\right)$ to $\left(\Sigma_{2}, \bullet_{2}\right)$.

As to digital, Maley maintains that "digital representation is the scheme we normally use to represent numbers. More explicitly, it is the representation of a number consisting of: a series of digits, where digit just means a numeral in a specific place; and a base, which is used to interpret the relative value of digits" (p. 8). This characterization can be reformulated as in the following definition.

Maley's definition of digital A representation is digital if and only if (i) $\Sigma_{1}$ is a set of numbers and $\Sigma_{2}$ is a set of numerals and (ii) the elements of $\Sigma_{2}$ have the form $d_{n} d_{n-1} \ldots d_{0}$, where $d_{n}, \ldots, d_{0}$ are digits, and, given a base $b$, they are interpreted as representing the number $\left(d_{n} \times b^{n}\right)+\left(d_{n-1} \times b^{n-1}\right)+\ldots+\left(d_{0} \times b^{0}\right)$.

According to Maley, in analog representations the represented elements $\Sigma_{1}$ are quantities, whereas in digital representations they are numbers. Hence, if Maley is right, the A/D distinction does not concern only the coding function from $\Sigma_{1}$ to $\Sigma_{2}$, but also the kinds of the represented things, i.e., the kinds of elements which are members of $\Sigma_{1} \cdot{ }^{14}$

\subsubsection{Some problems in Maley's account}

The examples of Section 2 are classified as follows according to Maley.

\begin{tabular}{|l|l|l|}
\hline MALEY CLASSIFICATION & classified cases & non classified cases \\
\hline analog representation & $(5),(6),(8),(10),(11)$ & $(2),(3),(4),(9),(12)$ \\
\hline digital representation & $(1),(7) ?$ & \\
\hline
\end{tabular}

Maley himself (p. 123, note 2) acknowledges that his definition of analog representation could be too strict: "It may be that requiring the function to be linear it is too strong: perhaps monotonicity would suffice. For example, a representation of earthquake intensity using logarithmic Richter scale might be a candidate for an analog representation". Hence, it seems to be appropriate to remove the condition that $\bullet_{1}$ and $\bullet_{2}$ must be additive from the definition of analog, and simply require that there must be a morphism from $\Sigma_{1}$ to $\Sigma_{2}$, that, for example, preserves the order between the involved quantities. So emended, the Maley's classification agrees entirely with the preliminary classification of analog cases. However, his characterization of digital representation is more problematic. The condition (i) of the definition of digital prescribes that the represented entities of a digital device can only be numbers. At face value, this seems to be at odds with the

\footnotetext{
${ }^{14}$ An anonymous referee noted that the definition of 'analog' at p. 123 of Maley (2011) deals with the representation of numbers, not quantities. This is true, but some lines below Maley writes of "the quantity to be represented" and some lines further of the representation of the "degrees of rotation", which is a quantity and not a number. Moreover in the footnote 2 of the same page Maley considers "a representation of earthquake intensity using the logarithmic Richter scale" as an example of analog representation. In general, Maley regards an analog representation as a "representation in which the represented quantity covaries with the representational medium" (p.122), indicating that Maley is thinking of a morphism between two sets of quantities.
} 
fact that texts, music, video, and so on can be digitally encoded. Maley deals with the problem by stating (p. 125 , note 6) that, although in his view it is true that a digital representation can represent only numbers, "the numbers represented can represent other things, such as characters, pixel values, or the time in the case of a digital clock". A problem undermines this thesis. While the states of bistable supports can be interpreted as the numbers 1 and 0 , this interpretation is not compelling at all, as the states of a bistable support might be represented, e.g., as + and -, or $O$ and $O$, and there is nothing in the nature of the states forcing them to be mapped on numbers. Just as an example, let us consider the representation of the color of a pixel by means of the states of a bistable support. To simplify matters, suppose that the pixel can only have three colors, e.g., (a certain shade of ) red, green and blue. Let the coding function be:

\section{$g:\{<$ red, OO $>,<$ green, OO $>,<$ blue, $O$ O $>\}$}

Maley would say that $g$ is not the actual coding function. Rather, the colors that pixels can take are associated with numbers, e.g., 0,1 and 2, and then these numbers are in turn associated with the states $0 \bigcirc$, ○, $\bigcirc$. But this reference to numbers adds nothing to the function $g$, which can be directly thought of as expressing the code of the system. Since in $g \Sigma_{2}$ is not a set of numerals, but simply the set of the possible states of a support, and $\Sigma_{1}$ is not a set of numbers, but a set of colors, the conditions (i) of the definition of digital seems to be too strict. More generally, in modern digital computers the bistable support can represent a set of numerals, but need not. That digital computers are digital only when they represent numbers seems to be an implausible consequence of Maley's view. If condition (i) is not correct, also condition (ii) falls away. Pace Maley, his view does not cover the example (9) of Section 2 (the representation of music by means of CDs) because the process of encoding music in CD pits and lands need not use numbers.

Another problem for Maley's account is that his definition of digital covers only a restricted class of numerical representations. For instance, the representation of integer positive and negative numbers (example 2) is not fully positional and therefore is not, strictly speaking, digital according to Maley's definition. This is also true of examples (3) (positional representation of rational numbers) and, especially, (4) (fractional representation of rational numbers). Similar remarks apply to the representation of a quantity by means of tables (example 12). As a consequence, Maley's view does not satisfies Desideratum 1, so that some improvements of his account are needed. ${ }^{15}$

\section{A new characterization of the $A / D$ distinction}

The characterization we are proposing of the $\mathrm{A} / \mathrm{D}$ distinction is focused on the coding function and originates from the observation that a basic condition must be fulfilled for a coding system to be useful. Whenever encoding and decoding are performed by different entities, either living beings or technological devices, the coding function $f_{\mathrm{c}}: \Sigma_{1} \rightarrow \Sigma_{2}$ must be completely and unambiguously communicable, and actually communicated, to the decoding entity, that is required to compute the function $f_{\mathrm{C}}{ }^{-1}$. This makes the explicit definition of $f_{\mathrm{C}}$ a critical aspect of the whole process of conveying information.

\footnotetext{
${ }_{15}$ Another scholar who dissents from the mainstream tradition and the common understanding is Blachowicz (1997). He denies the equivalences digital=discrete and analog=continuous, and suggests that the $\mathrm{A} / \mathrm{D}$ distinction should be accounted for in a different way. He states that, in a digital system, a symbol stands for a type, while in an analog system the representation and the represented object are tokens of the same type. While this characterization implies that in an analog system the representation and the represented object have some sort of structural resemblance (which is one of our claims), the hypothesis that the type/token distinction can be the basis for the A/D distinction is critical. In fact, as shown in Section 2.1, the coding function always maps types into types and, as we shall see, this is true both for analog and digital representation.

Some of these remarks apply also to the characterization of Dretske (1981). He thinks that a signal is digital if it does not carry more information than required by the aims of the system, analog if it carries more specific information than required. For instance, suppose that the aim of a system is to activate a brake when a vehicle exceeds $70 \mathrm{~km} / \mathrm{h}$; 列e system is made up of a speedometer capable of registering the vehicle speed and a converter which generates a signal when the registered speed exceeds $70 \mathrm{~km} / \mathrm{h}$. Dretske claims that the speedometer carries analog information because it is more specific than required by the system (the only important thing is whether the speed exceeds or $70 \mathrm{~km} / \mathrm{h}$ or not; it is not important whether the speed is 56 or $57 \mathrm{~km} / \mathrm{h}$ ), while the signal carries digital information. In converting the information from the analog to the digital format there is a loss that, however, is compensated by the fact that the digital signal provides a classification of the significant ranges of the input variable: every speed under $70 \mathrm{~km} / \mathrm{h}$ is collected in one class and every speed over $70 \mathrm{~km} / \mathrm{h}$ in another class. But, again, every coding function maps tokens into types: the speedometer is not an exception because it maps every speed in some class (the class of $1 \mathrm{~km} / \mathrm{h}$, the class of $2 \mathrm{~km} / \mathrm{h}$, etc.). Furthermore, it is hard to see how an analog clock, a paradigmatic example of analog device, carries more information than required by the aim of the system. Actually, it is not even clear whether Dretske wishes to provide a new characterization of the common A/D distinction or to put forward a definition of a different distinction: he says that he is using "the familiar terminology - analog vs digital - in a slightly unorthodox way" (p. 137).
} 


\subsection{Intensional vs extensional definitions as coding strategies}

It is well known that two general strategies are available to define a set, and therefore specifically a function $f: \mathrm{A} \rightarrow \mathrm{B}$ thought of as the subset of the set $\mathrm{A} \times \mathrm{B}$ of the ordered pairs $\langle a, b\rangle$ such that $b=f(a)$. Indeed, a coding function $f$ can be defined:

- by listing a set of conditions on $\Sigma_{1} \times \Sigma_{2}$ that are necessary and sufficient to determine whether $\sigma_{2}=f_{\mathrm{C}}\left(\sigma_{1}\right)$ for any ordered pair $\left\langle\sigma_{1}, \sigma_{2}\right\rangle$; this is called an intensional definition;

- by listing the set of all the ordered pairs $\left\langle\sigma_{1}, \sigma_{2}\right\rangle$ such that $\sigma_{2}=f_{\mathrm{C}}\left(\sigma_{1}\right)$; this is called an extensional definition. ${ }^{16}$

Before analyzing these strategies and comparing them in their features and applicability to coding systems, let us introduce a simple example. A teacher needs to communicate the marks, let us suppose integer numbers in the range $\Sigma_{1}=\{1,2, \ldots, 10\}$, that some students achieved in an examination. Aiming at making the students aware of the complexities of the process of conveying information, the teacher decides not to perform the task by the customary, verbal or written, means of communication. Instead, she adopts alternatively two exemplary, although peculiar, solutions for communicating the marks $\sigma_{1} \in \Sigma_{1}$ :

Solution 1: the teacher encodes each mark $\sigma_{1}$ by means of a proportional amount of water, say $k \sigma_{1}$ for a given $k>0$ (hence, $\sigma_{2}=f\left(\sigma_{1}\right)=k \sigma_{1}$; for the sake of simplicity measurement units are omitted) (the writing process); by receiving a given amount of water (the transmission process), each student obtains the quantity value $\sigma_{2}$ by means of measurement ${ }^{17}$ (the reading process) and finally he ascertains the assigned mark by decoding such quantity value, i.e., by computing $f^{-1}\left(\sigma_{2}\right)=\sigma_{2} / k$;

Solution 2: the teacher encodes each mark $\sigma_{1}$ by means of a distinct shape $\sigma_{2}$, thus assuming the correspondences mark $1 \leftrightarrow$ shape 1 , mark $2 \leftrightarrow$ shape $2, \ldots$, (the writing process); by receiving a given object (the transmission process), each student recognizes its shape (the reading process) and finally he ascertains the assigned mark by decoding the shape, i.e., by computing $f^{-1}\left(\sigma_{2}\right)$.

It is easy to prove that both solutions satisfy the three conditions that define a coding system $\left\langle\Sigma_{1}, \Sigma_{2}, f_{\mathrm{C}}\right\rangle$ :

i) $\Sigma_{1}$ is the range of a classification function, in this case a map from examination tests to marks;

ii) $\Sigma_{2}$ is a set of instantiable objects, in this case either given amounts of water (Solution 1) or given shapes of objects (Solution 2);

iii) $f_{\mathrm{C}}$ is bijective.

Hence, provided that students are informed of the adopted coding function, in principle both solutions produce correct results, i.e., are able to convey unambiguous information. Yet, such solutions are structurally different, as they imply:

- the intensional definition of the coding function in Solution 1;

- the extensional definition of the coding function in Solution 2.

This gives a hint on the general strategies for the communication of a coding system to receivers (students in this case), and shows the greater efficiency of Solution 1, which exploits a single parametric expression such as $\sigma_{2}=k \sigma_{1}$, compared to Solution 2, which instead requires the function to be expressed as a list of instances of the same pattern: mark $\sigma_{1} \leftrightarrow$ shape $\sigma_{2}$.

To further emphasize the different features of the two solutions, a second issue can be taken into account: what changes are required in the coding system if the set of objects to be represented is extended, e.g., to include not only integer, but also fractional marks as $1 / 2,1 \frac{1}{2}, 2 \frac{1}{2}$, etc.? Whenever the capability to

\footnotetext{
$\overline{16}$ This clear-cut characterization hides some subtle issues that, although not specifically critical for our analysis, can be mentioned. The listing of the pairs $\langle a, b\rangle$, i.e., the extensional definition of the function, is achievable in two different ways: 1) by explicitly listing, for each pair, both the first and the second element; 2) by explicitly listing, for each pair, only the second element, and then giving a property which the elements of the domain of the function are to satisfy in order to be mapped to an element in the list. Whereas the first way is only possible when the function domain is finite, the second way is also possible when the domain is infinite. An example of the first form of the definition is: $b=f(a)$ if and only if $(a=1$ and $b=1)$ or $(a=2$ and $b=2)$ or $(a=3$ and $b=1)$ or ( $a=4$ and $b=2$ ). An example of the second form is: $b=f(a)$ if and only if ( $a$ is odd and $b=1)$ or ( $a$ is even and $b=2)$. Moreover, the intensional strategy is the only one available when the image of the function is an infinite set, while the extensional strategy is the only one available when the image of the function is an extensionally defined set and the function is one to one.

${ }^{17}$ Of course the student must be able to discriminate between the different possible quantities of water he can receive. In particular, he must be equipped with an instrument able to distinguish $k \cdot n$ of water from $k(n+1)$ of the same substance.
} 
discriminate between given amounts of water (i.e., the resolution of the involved writing and reading devices, in this case controlled by the value of the multiplicative factor $k$ ) remains sufficient, Solution 1 can be maintained without any change. On the other hand, Solution 2 requires the introduction of new shapes and correspondingly the extension of the coding function. Therefore, also from this point of view, Solution 1 appears to be more efficient than Solution 2.

At this stage of the analysis, one could assume Solution 1 and Solution 2 to be representative of that which could be called "continuous coding" and "discrete coding" respectively. To explore the soundness of this hypothesis, and to show that it is in fact wrong, let us introduce a third possible solution to the problem presented in the example. For communicating the marks:

Solution 3: the teacher encodes each mark $\sigma_{1}$ by means of a proportional number of empirically identical objects (e.g., pebbles), say $k \sigma_{1}$ for a given integer $k>0$ (hence $\sigma_{2}=f_{\mathrm{C}}\left(\sigma_{1}\right)=k \sigma_{1}$ ) (the writing process); by receiving a given number of pebbles (the transmission process), each student obtains the quantity value $\sigma_{2}$ by counting (the reading process) and finally he ascertains the assigned mark by decoding such quantity value, i.e., by computing $f_{\mathrm{C}}^{-1}\left(\sigma_{2}\right)=\sigma_{2} / k$.

With respect to both the issues of communicating the coding system and changing it to extend the set of objects to be represented, Solution 3 appears to be structurally similar to Solution 1 as far as the factor $k$ is chosen so to avoid the loss of resolution due to the coarse quantization of the support adopted in the case of Solution 3 (e.g., at least $k=2$ if the elements of the set $\left\{1 / 2,1,1 \frac{1}{2}, 2, \ldots, 10\right\}$ must be represented). This shows that the difference between Solution 1 and Solution 2 is not related to the only apparent fact that the former adopts a continuous coding and the latter a discrete one. Indeed, despite its structural identity to Solution 1 , Solution 3 adopts a paradigmatically discrete support. What is relevant here is not the continuous vs discrete distinction, but the strategy adopted to define the coding system: according to an intensional definition in the case of Solutions 1 and 3, and according to an extensional definition in the case of Solution 2.

The greater efficiency of Solutions 1 and 3 compared to Solution 2 also appears in the fact that the former can be applied even when the cardinality of the set of objects to be encoded is not known in advance (with the only condition, as stated above, on the resolution of the coding function), whereas the latter requires such set to be finite and extensionally known.

As a partial conclusion, the strategy based on intensional definitions has several advantages compared to the one based on extensional definitions. On the other hand, the former is not unconditionally the best one, as the intensional definition of a coding function implies the presence of a structure on the function domain, a requirement that can be avoided in the case of extensional definitions. This condition is so important for our analysis that it is worth some further consideration.

\subsection{Structures as facilities, structures as constraints}

As already pointed out, the intensional definition of a function $f: \mathrm{A} \rightarrow \mathrm{B}$ consists of a set of conditions that are necessary and sufficient to identify the value $f(a) \in \mathrm{B}$ for any argument $a \in \mathrm{A}$. Such conditions can only be expressed if the function domain $\mathrm{A}$ is equipped with a structure to be exploited in the definition itself. For example, if $\mathrm{A}$ is linearly ordered then a function could be intensionally defined as "for a given element $a^{\prime} \in \mathrm{A}$, map all $x<a^{\prime}$ to $0, a^{\prime}$ to $1 / 2$ and all $x>a^{\prime}$ to 1 ". This example also shows that intensional definitions generally do not impose a structure on the function range (consider reformulating the three cases by mapping to, e.g., "Paris", "London", and "Rome"). On the other hand, the functions taken into account here are part of a coding system, and therefore they must be bijective. It should be obvious that the intensional definition of a bijective function implies the presence of a structure also on the function range: since the number of atomic conditions in the definition must be less than the cardinality of the function domain, and the cardinality of the function domain and range is the same because of the bijectivity, then the conditions must be able to identify subsets of the range, a situation that is possible only in the presence of a structure on the range itself. Hence, the intensional definition of a coding system $\left\langle\Sigma_{1}, \Sigma_{2}, f_{\mathrm{C}}\right\rangle$ implies that both the domain $\Sigma_{1}$ and the range $\Sigma_{2}$ of the coding function $f_{\mathrm{C}}$ are equipped with a structure. This introduces a further constraint: since, by definition, the elements of $\Sigma_{2}$ must be instantiable, i.e., there exists a set $\mathrm{O}_{2}$ of empirical objects such that they are tokens whose types are the objects of $\Sigma_{2}$, a coding system is intensionally definable only if the set $\mathrm{O}_{2} / \approx$ of the classes of $\approx$-equivalence of such tokens is, in its turn, equipped with a structure that makes it isomorphic to $\Sigma_{2}$, being $\approx$ the relation of empirical indistinguishability in the writing/reading process. This is 
easily verified, in particular, in the case of Solutions 1 and 3 above: the classes of equivalent amounts of water and of equivalent numbers of pebbles are linearly ordered by the empirical relations 'greater amount' and 'greater number'. On the contrary, the tokens adopted in Solution 2 do not fulfill this constraint (no ordering among the shapes need to be determined), that, however, is not mandatory since in this case the definition of the coding system is extensional.

The following table synthesizes the conclusions reached so far, by comparing two strategies of definition of coding systems in terms of four criteria.

\begin{tabular}{|l|l|l|l|l|}
\cline { 2 - 5 } \multicolumn{1}{c|}{} & $\begin{array}{l}\text { Criterion 1: how to de- } \\
\text { fine/communicate } \\
\left\langle\Sigma_{1}, \Sigma_{2}, f_{\mathrm{C}}\right\rangle ?\end{array}$ & $\begin{array}{l}\text { Criterion 2: how to ex- } \\
\text { tend }\left\langle\Sigma_{1}, \Sigma_{2}, f_{\mathrm{C}}\right\rangle ?\end{array}$ & $\begin{array}{l}\text { Criterion 3: must } \Sigma_{1} \\
\text { have a structure? }\end{array}$ & $\begin{array}{l}\text { Criterion 4: must } O_{2} / \approx \\
\text { have a structure making } \\
\text { it isomorphic to } \Sigma_{2} ?\end{array}$ \\
\hline $\begin{array}{l}\text { Intensional } \\
\text { definitions }\end{array}$ & $\begin{array}{l}\text { by a parametric expres- } \\
\text { sion }\end{array}$ & (nothing required) & yes & yes \\
\hline $\begin{array}{l}\text { Extensional } \\
\text { definitions }\end{array}$ & $\begin{array}{l}\text { by a list of instances of } \\
\text { the same pattern }\end{array}$ & $\begin{array}{l}\text { by explicitly listing the } \\
\text { new instances }\end{array}$ & no & no \\
\hline
\end{tabular}

As the last step of our analysis, let us point out that intensional definitions of coding systems can be classified into two general categories, depending on whether the coding function $f_{\mathrm{C}}$ preserves the structure defined on its domain or not. In the former case, for each relation $R_{\Sigma 1}$ on $\Sigma_{1}$ (let us consider binary relations for the sake of simplicity) there is a relation $R_{\Sigma 2}$ on $\Sigma_{2}$ such that $R_{\Sigma 1}\left(\sigma_{1, i}, \sigma_{1, j}\right)$ if and only if $R_{\Sigma 2}\left(f_{\mathrm{C}}\left(\sigma_{1, i}\right), f\left(\sigma_{1, j}\right)\right)$. Hence, $f_{\mathrm{C}}$ is an isomorphism between $\Sigma_{1}$ and $\Sigma_{2}$. On the other hand, coding functions are not generally constrained to preserve the structure defined on their domain. As a simple example, given $\Sigma_{1}=\Sigma_{2}=$ $\{0,1, \ldots, n-1\}$ for $n>1$, let us consider the function $f(i)=i+1 \bmod n$, i.e., in the case $n=3$, the function $f(0)=1$, $f(1)=2, f(2)=0$. Of course, this function is (defined in intensional way and is) bijective but does not preserve the ordering on the natural numbers, i.e., in this sense it is not an isomorphism on $\{0,1, \ldots, n-1\}$ into itself. This shows that, in general, three strategies to define coding systems are available:

- extensional;

- isomorphic intensional;

- non-isomorphic intensional.

\subsection{Analog and digital as features of coding systems}

The reader can now easily guess the characterization of the A/D distinction we propose: 'analog' and 'digital' are strategies adopted to define coding systems, and:

- a coding function is analog if and only if it is defined in an isomorphic intensional way;

- a coding function is digital if and only if it is defined in an extensional way,

where a function is defined in an extensional way when its definition is based on a correspondence determined by a list. The definitions of 'analog' and 'digital' become:

Definition A: analog = pertaining to the representation of information based on an isomorphic intensional coding function.

Definition $D$ : digital $=$ pertaining to the representation of information based on an extensional coding function.

As introduced above, Solution 2 is an example of digital coding: no structure is available on the given set of shapes, so that the coding function can be defined only by the exhaustive (then unavoidably finite, hence, in particular, discrete) listing of the ordered pairs 〈represented class, representing type〉. Solutions 1 and 3 are instead examples of analog coding: the structure available on the given sets of amounts of water / number of pebbles is critically exploited in the isomorphic mapping of the represented class into the representing type. This shows that being finite and discrete are necessary conditions of being digital, but they could be not sufficient, as Solution 3 (discrete but analog) shows. ${ }^{18}$

\footnotetext{
${ }_{18}$ Analog coding is characterized by what O'Brien \& Opie (2006) call "second order resemblance". According to these scholars first order resemblance concerns two or more objects sharing the same physical properties, e.g., the same mass. Second order resemblance concerns two or more symbols (the authors speak of "representing vehicles") having some relations mirrored by the objects that
} 
The above definitions only require for the information representation to be based on an extensional / isomorphic intentional coding function. Indeed, in many cases, and typically when the set of entity to be represented is both discrete and denumerable, the coding function is recursively defined. If the base case of a recursive definition is given by specifying a list of pairs, then the coding function is to be still considered as digital, since there is no similarity between the set of signs introduced by the definition and the set of designed entities. As a consequence, the representation of discrete numerical entities, such as integer numbers, dense numerical entities, such as rational numbers, and, in general, data types, turns out to be digital. $^{19}$

Remark. Some functions can be defined both intensionally and extensionally, and in particular, any finite, intensionally defined function can be also defined extensionally. Thus, some devices can be considered analog or digital depending on how the coding function they realize is defined. Indeed, it is the actual rule that communicating agents use to encode and decode information that determines the nature of the code, so that a device is analog or digital depending on the actual definition of the function the agents use to encode pieces of information on the support. This does not seem to be a drawback. The A/D distinction concerns the code used to link signs to their meanings: since a code exists only if there are some agents that use the code, it is the actual code used by the agents which determines if a device is analog or digital.

The examples of Section 2 are classified as follows according to our account.

\begin{tabular}{|l|l|l|}
\hline OUR CLASSIFICATION & classified cases & Non-classified cases \\
\hline analog representation & $(5),(6),(8),(10),(11)$ & none \\
\hline digital representation & $(1),(2),(3),(4),(7),(9),(12)$ & none \\
\hline
\end{tabular}

Our classification satisfies desideratum 1 because all cases are classified. In addition, our proposal conforms to the preliminary classification and to the A1, A2, D1, D2 principles, also satisfying desideratum 2. In fact, if the coding function is extensional, i.e., it is defined by explicitly listing pairs of elements, then the range of the function has to be finite, and therefore discrete, otherwise the list could not be produced. Furthermore, the only constraint to be fulfilled on the function range is that different elements of the domain are to be associated to different elements in the range, so that the way in which the elements are associated to the signs is wholly conventional. On the contrary, if the coding function is intensional, than the relevant structure of

symbols refer to. One of their examples is that of a mercury thermometer used to represent temperature in virtue of the linear relationship between the length of a column of mercury and ambient temperature. O'Brien and Opie claim that analog computers use symbols that have a second order resemblance with the objects they refer to (p. 4). Moreover, they believe that one of the differences between classical computation systems and connectionist systems is that the latter use representing vehicles showing structural resemblance with the domain they represent. If this is correct, connectionist systems would be analog systems according to our definition and it would be possible to characterize the difference between classical computation systems and connectionist systems in terms of the $\mathrm{A} / \mathrm{D}$ distinction. Thanks to an anonymous referee for drawing our attention to this paper.

${ }^{19}$ To illustrate, let us consider the coding function on which positional systems are based. The very concept of a positional system is grounded on a set of instructions: by an intensional rule it is easily and efficiently learned that the numeral $d_{n} d_{n-1} \ldots d_{0}$, where each $d_{i}$ is a digit (so that, e.g., in ' $314^{\prime}$ ' 3 ' $=d_{2}$, ' 1 ' $=d_{1}$, and ' 4 ' $=d_{0}$ ), stands for the number $\Sigma d_{i} b^{i}$, where $b$ is the base of the adopted number system (hence, $3 \cdot 10^{2}+1 \cdot 10^{1}+4 \cdot 10^{0}$, if $b=10$ ). In this sense, the positional system is in fact a hybrid one: it is extensional in the coding function for the basic digits and intensional in the coding function for sequences of digits. Something similar seems to be at work in the case of languages. As formal languages are usually compositional, there is an isomorphism between syntax and semantics: each time two symbols are linked together, the corresponding meanings are composed, so that a complex meaning corresponds to the composition of symbols. This part of the coding function can be considered intensional: there is an analogy between the complex signs and the complex meanings they encode because the signs have the same structure as their meanings. The isomorphism between syntax and semantics of natural languages is one of the basic assumptions of the so-called "Montague grammars" (see Montague (1974)). Anyway, in such cases, the essential part of the definition of the coding function is given by the choice of the set of basic symbols: the correspondence between basic symbols and numbers is conveyed by means of a list and the number of basic symbols determines the base of the positional system Conversely, coding systems with an intensional isomorphic base are also possible. Surprisingly, analog clocks are systems of this kind. Naturally, a morphism between the angle covered by the hand and the time are exploited by analog clocks, but if two or more hands are present, there are conventions that are not part of this morphic base. For instance, that the shorter hand (or the hand of a certain color) indicates the hours while the other one the minutes is a piece of information not coded by the base. The coding function $<<$ shorter hand, hours $>,<$ longer hand, minutes $>>$ is not grounded on any isomorphism. 
the domain has to be preserved in the range. Hence, the relations between the elements of the domain have to be mirrored by the relations between the elements of the range, so that the correlation between variations in the domain and variation in the range follows. Therefore, this characterization is able to explain why the coding systems, and the related devices, that are considered to be analog are indeed "analog", as, according to this interpretation, analog coding implies the existence of an analogy, actually an isomorphism, between $\Sigma_{1}$ and $\Sigma_{2}$ and, consequently, between types and tokens to be transmitted. ${ }^{20}$

In addition, this characterization is also able to account for the advantages and drawbacks of either coding strategy. When the cardinality of the set $\Sigma_{1}$ of objects to be represented is high (a common situation in real cases involving, e.g., audio or video information), analog coding is much simpler, whereas digital coding is more flexible as it does not require the presence of any structure either in the information to be encoded or in the adopted support. This suggests a simple and consistent interpretation to understand why digital is today commonly preferred over analog, whereas practically all telecommunication systems were first designed and implemented on the basis of analog coding techniques (an exception is telegraph): the increasing computational power and memory storage capability of the available technological devices have overshadowed the fact that digital processes require far more resources than comparable analog ones, thus making the flexibility of digital coding cheaply and widely available.

Finally, let us consider how our characterization of the A/D distinction relates to the ones adopted by the authors discussed above. On the one hand, it is not difficult to see that our account is in accordance with them in classifying most of the central cases of A/D representation, even though the classification criteria are different. This is due to the fact that most of the criteria adopted by the other authors, concerning either the domain, or the codomain, or the implementation of the coding function, follow from our definition, which is focused on the function itself. Thus, the discreteness of the domain and the possibility of having reliable writing / reading processes are accounted by the fact that the coding function is extensionally defined, while the continuity of the codomain and the possibility of having unreliable writing/reading processes are accounted by the fact that the coding function is intensionally defined. Hence, the original point of our characterization, focused on the coding function, allows us to account for the success of the other ones in classifying most of the paradigmatic cases. On the other hand, it has also to be highlighted what our characterization implies as for the two basic distinctions variously exploited by the other authors (i) continuous / discrete and (ii) unreliable / reliable coding.

(i) As to the correspondence $\mathrm{A} / \mathrm{D} \leftrightarrow$ continuous/discrete, the conclusion is that while digital coding, which requires the coding function to be defined by explicitly listing its instances, is finite and therefore in particular discrete, at least in some cases also analog coding can be discrete.

(ii) As to the correspondence $\mathrm{A} / \mathrm{D} \leftrightarrow$ unreliable/reliable, our account implies that the reliability of encoding and decoding processes, which is indeed a critical experimental feature, is in principle unrelated to the digital strategy of coding. It is a correct observation that digital systems are usually more reliable than analog ones, but this is an operative matter, relative to the way in which the coding function is implemented, not to the way in which it is defined. ${ }^{21}$

\section{Conclusions}

In this paper the A/D distinction has been referred to coding systems, which can be defined according to either an intensional or an extensional strategy: under the hypothesis of isomorphic mapping the former is analog, the latter digital. On the other hand, the condition for the encoded information to be instantiated makes this interpretation relevant also to physical systems, thus accounting for the common understanding that being analog or digital has something to do with such systems as far as they are adopted as support for

\footnotetext{
${ }^{20}$ For example, vinyl audio records are analog devices because their configurations (sequences of depths of the groove) are isomorphic to the acoustic power and frequency of the stored sound. On the contrary, there is no isomorphism between the configurations of a digital device, such as the pits and lands of a $\mathrm{CD}$, and the music recorded on it. Rather, it is exactly the extensional coding system exploited to write and read CDs that makes them such versatile supports, able to store not only music but also much less structured information such as texts, software, and so on.

${ }^{21}$ It is straightforward to show that reliability is unrelated to our characterization of analog and digital coding. In reference to the problem of communicating the marks that some students achieved in an examination, the reliability of (analog) Solution 1 , where the mark $\sigma_{i}$ is communicated by $k \sigma_{i}$ of water, depends on the factor $k$, and the greater $k$ the greater the reliability. On the other hand, the reliability of (digital) Solution 2, where the mark $\sigma_{i}$ is communicated by the $i$-th shape, might be hindered by shapes that are insufficiently different with one another. Furthermore, in both cases the reliability can be increased by replicating the transmission (the mark $\sigma_{i}$ is communicated by 2 , or more generally $n$, samples of $k \sigma_{i}$ of water; and by 2, or more generally $n$, objects of shape $i$ ), highlighting that the trade-off is between reliability and amount of resources devoted in the transmission, not between reliability and A/D.
} 
information. According to this characterization, both continuous and discrete supports can be used in analog coding, whereas digital coding must exploit discrete supports because any extensional definition can be realized only on finite domains. The intuition is preserved here that the basic character of analog representations is constituted by the structural resemblance between what is represented and the representing signs. As we have seen, our account satisfies the stated desiderata and gives some insights on the possibility for analog devices of not being based on physical quantities ${ }^{22}$ and for digital devices of not being based on numbers / numerals. In synthesis, the benefits of this proposal are at least that:

1) the $\mathrm{A} / \mathrm{D}$ distinction turns out to be irreducible to the continuous / discrete one, and therefore its importance is maintained and highlighted;

2) the A/D distinction turns out to be a characterization of the strategies to represent information, and not of the kinds of signals or supports of the representation, and the conditions that make analog and digital representations possible are clearly identified; ${ }^{23}$

3) the $A / D$ distinction turns out to be independent of the current physical theories concerning the fine structure of the world. ${ }^{24}$

Finally, the characterization criterion we have proposed, related to the way coding functions are defined and communicated, leads to the unambiguous classification of representation strategies that includes both hybrid, partly analog and partly digital, strategies, and a third coding strategy, neither analog nor digital, based on non-isomorphic intensional mappings, as is typical of cryptographic codes, a case of "hidden communication". 25

\section{References}

Adriaans, P. \& van Benthem, J. (eds.) (2008), Philosophy of Information (Handbook of the Philosophy of Science). London: Elsevier.

Allen, R. \& Mills, D. (2004). Signal Analysis: Time, Frequency, Scale, and Structure. IEEE Computer Society Press.

Barwise, J. (1989). The Situation in Logic. Stanford, CA: CSLI lecture notes.

Barwise, J. \& Seligman, J. (2007). Information Flow: The Logic of Distributed Systems. Cambridge: Cambridge University Press.

Bateson, G. (1979). Mind and Nature: A Necessary Unity. New York: Dutton.

Blachowicz, J. (1997). Analog Representation Beyond Mental Imagery. Journal of Philosophy. 94, 55-84.

Bremer, M. \& Cohnitz, D. (2005). Information and Information Flow: An Introduction. Hausenstamm: Ontos Verlag.

Burda, I. (2005). Introduction to Quantum Computation. Boca Raton: Universal Publishers.

Carnap, R. \& Bar-Hillel, Y. (1953). Semantic Information. The British Journal for the Philosophy of Science. $14,147-157$.

Collins, H. (2010). Tacit and Explicit Knowledge. Chicago: The University of Chicago Press.

Dretske, F. (1981). Knowledge and the Flow of Information. Cambridge (Mass.): MIT Press.

Floridi, L. (2011a). The Philosophy of Information. New York: Oxford University Press.

Floridi, L. (2011b). Semantic Conceptions of Information. In Stanford Encyclopedia of Philosophy, http://plato.stanford.edu/entries/information-semantic.

Goodman, N. (1968). Languages of Art: An Approach to the Theory of Symbols. Indianapolis: The Bobbs-Merrill Company, Inc.

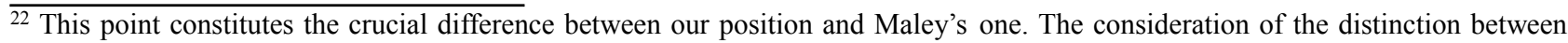
intensionally and extensionally defined function can be seen as the most intuitive way to generalize Maley's position so to cover the paradigmatic examples introduced in Section 2.

${ }^{23}$ Bar charts, which are typically considered analog representations of quantitative information, do not become digital only because they are displayed on discrete devices, like LCD computer screens, whose elements are pixels. Nothing is gained by claiming that pixels are invisible at certain distance from the screen and that are not thereby part of the representation: that a representation is analog at a certain distance but becomes digital if observed more closely would be a peculiar consequence of defense attempt.

${ }^{24}$ Since the current best physical theory of the fine structure of the matter is quantum mechanics, we should conclude that all devices are ultimately digital, and therefore that analog devices are only "ideal systems": this seems a serious drawback for traditional accounts.

${ }^{25}$ In fact cryptographic codes are usually defined intensionally, but they avoid any structural similarity between the original message and the cyphered one, for the obvious reason that analogies might be detected and then exploited to decipher the message even without knowing the key.
} 
Hartley, R. (1928). Transmission of Information. Bell System Technical Journal. July, 535-563.

Haugeland, J. (1981). Analog and analog. Philosophical Topics. 12, 213-222.

IEV International Electrotechnical Commission, IEC 60050 series, also as Electropedia. The World's Online Electrotechnical Vocabulary. http://www.electropedia.org.

Katz, M. (2008). Analog and Digital Representation. Minds \& Machines. 18, 403-408.

Lewis, D. (1971). Analog and Digital. Nous, 5, 321-327.

Luecke, J. (2005). Analogue and Digital Circuits for Electronic Control System Applications. Burlington-Oxford: Elsevier.

Maley, C. (2011). Analog and Digital, Continuous and Discrete. Philosophical Studies, 155, 117-131.

Minsky, M. (1972). Computation: finite and infinite machines. London: Prentice-Hall Int.

Montague, R. (1974). Formal Philosophy. New Haven and London: Yale University Press.

O’Brien, G. \& Opie, J. (2006). How do Connectionist Networks Compute? Cognitive Processing. 7, 30-41.

Perry, J. (1997). Indexicals and Demonstratives, in R. Hale \& C. Wright (eds.), A Companion to the Philosophy of Language, Oxford: Blackwell, pp. 586-612.

Piccinini, G.\& Scarantino A. (2011). Information Processing, Computation, and Cognition. Journal of Biological Physics, 37, 1-38.

Shannon, C. (1948). A Mathematical Theory of Communication. Bell System Technical Journal. 27, 379-423 \& 623-656. 